\title{
Effects of Choline, Betaine, and Wheat Floral Extracts on Growth of Fusarium graminearum
}

\author{
Jessica S. Engle, Graduate Research Associate, Patrick E. Lipps, Professor, Terry L. Graham, Professor, and \\ Michael J. Boehm, Associate Professor, Department of Plant Pathology, The Ohio State University, Wooster 44691
}

\begin{abstract}
Engle, J. S., Lipps, P. E., Graham, T. L., and Boehm, M. J. 2004. Effects of choline, betaine, and wheat floral extracts on growth of Fusarium graminearum. Plant Dis. 88:175-180.

Fusarium head blight has been more severe when infection occurs during anthesis, indicating that floral organs may be important infection courts. Choline acetate and glycinebetaine have been extracted from wheat and reported to be growth stimulants of Fusarium graminearum. They are hypothesized to enhance infection and tissue colonization. Growth of $F$. graminearum was examined on media amended with extracts from floral parts of nine wheat genotypes with various Fusarium head blight resistance levels. Results indicated no significant effect of anther, palea, or lemma extracts on radial growth when compared with unamended controls. Effects on spore germination and hyphal growth of $F$. graminearum by choline, betaine, and an equimolar mixture at concentrations ranging from 0.01 to $1,000 \mu \mathrm{M}$ also were examined. Spore germination was not significantly $(P \leq 0.05)$ affected by choline, betaine, or a combination of the compounds compared with unamended controls. Radial hyphal growth also was not consistently affected $(P \leq 0.05)$ by choline or betaine when compared with controls. Equimolar mixtures of the two compounds showed significant slight reduction in growth rate at higher concentrations when compared with controls. The reduction in growth rate was due to higher concentrations of betaine. Results of this study indicate that endogenous compounds in floral parts may not be associated with wheat resistance to $F$. graminearum.
\end{abstract}

Additional keywords: Gibberella zeae, Triticum aestivum, winter wheat

Fusarium head blight of wheat (Triticum aestivum L.), caused by Fusarium graminearum Schwabe (teleomorph: Gibberella zeae (Schwein.) Petch), has become a widespread problem in the United States as a result of increased implementation of reduced-tillage farming practices, especially in crop rotations where wheat follows corn (Zeae mays L.) $(4,8,10)$. F. graminearum infection of the floret causes sterility, poor seed fill, poor seed quality, and contamination of grain with the mycotoxin deoxynivalenol, which has resulted in widespread economic loss in the northcentral region of the United States $(2,4,5,17,22)$. Minimal control of Fusarium head blight has been achieved through fungicide application and cultural practices to date, making planting of moderately resistant cultivars the primary management tool $(2,4,11-13,16,17)$.

Corresponding author: P. E. Lipps

E-mail: lipps.1@osu.edu

Salaries and research support were provided by state and federal funds appropriated to the Ohio Agricultural Research and Development Center, the Ohio State University. Partial funding for this research was obtained from the United States Wheat and Barley Scab Initiative.

Accepted for publication 9 September 2003.

Publication no. D-2003-1118-04R

(C) 2004 The American Phytopathological Society
Fusarium head blight severity levels in the field are highest when wet weather coincides with wheat anthesis and in the greenhouse when anthers, rather than emasculated spikelets, are inoculated $(1,3,11,16,17,19,24)$. These findings suggested that anthers are the common route of entry into the plant and that there may be compounds in anthers that stimulate growth of $F$. graminearum (24).

In an effort to determine components of resistance to $F$. graminearum, several researchers have examined the effect of compounds in anthers on growth of $F$. graminearum $(15,24)$. In one study, anther extracts from a susceptible genotype stimulated growth of $F$. graminearum in vitro to a greater extent than extracts from other wheat plant structures (24). In contrast, another group of researchers found that extracts from anthers of a highly resistant cultivar significantly stimulated mycelial growth, but extracts from anthers of a susceptible cultivar did not (15). These conflicting results indicate that the nature of the interaction between endogenous compounds in floral structures and $F$. graminearum infection may be more complex than hypothesized. These two studies used different protocols for extraction and determining the effects of floral compounds on $F$. graminearum, making direct comparison of results questionable $(15,24)$. For example, the genotypes evaluated were different and protocols for extraction of the floral compounds also differed. Additionally, only one isolate of $F$. graminearum was used in each study. These differences may have contributed to variation in reported results.

Several researchers have extracted compounds from wheat anthers, namely choline acetate and glycinebetaine, which were thought to be responsible for fungal growth stimulation $(18,23,24,26)$. Additionally, $F$. graminearum has been shown to possess separate constitutive high-affinity transport systems that are specific for both choline and betaine $(20,21)$, indicating that choline and betaine may be specifically utilized by $F$. graminearum. Although the reason the fungus utilizes these compounds remains unknown, researchers have examined the effect of choline and betaine in vitro with results of both stimulation and reduction of $F$. graminearum hyphal growth $(15,24)$. The assay used in previous experiments involved cutting wells in the agar layer in petri dishes with cork borers, drying the agar, and then placing a solution containing the compound in a central well and measuring radial growth from inoculated neighboring wells toward the central well $(15,24,27)$. It was likely that a concentration gradient formed in the solid medium between the central well and the adjacent inoculated wells, affecting growth of the fungus. Additionally, varying concentrations of floral compounds may have different effects on spore germination than on radial growth.

The nature of resistance in wheat to $F$. graminearum has yet to be determined, but preformed compounds in floral parts may play a major role in fungal infection and colonization. Previous studies $(15,18,23$, $24,26)$ were conducted on a limited range of compound concentrations and limited wheat genotypes. Thus, the first objective of this study was to examine the effects of choline, betaine, and an equimolar mixture of both compounds on hyphal extension and spore germination of $F$. graminearum over a larger range of concentrations using a protocol that would limit a concentration gradient. The second objective was to determine the effects of extracts of different floral structures from nine wheat genotypes with varying resistance reactions to $F$. graminearum on hyphal growth. In contrast to a previous work (15) that tested one susceptible and one resistant genotype, we tested several genotypes expressing a range of resistance reactions in the field to 
provide more complete information on the growth response of $F$. graminearum.

\section{MATERIALS AND METHODS}

Hyphal extension. Three $F$. graminearum isolates characterized by high aggressiveness on the susceptible spring wheat $\mathrm{cv}$. Wheaton were obtained in Ohio from infected wheat and were selected based on their ability to produce abundant perithecia in pure culture. To examine the effects of choline and betaine on radial growth, solid media ( $2 \%$ water and dextrose agar) were amended to obtain final concentrations of: $0.01,0.1,1.0,10,100$, and $1,000 \mu \mathrm{M}$ of choline chloride (Sigma-Aldrich, St. Louis), betaine hydrocloride (Sigma-Aldrich), and an equal molar concentration of the two compounds. Compounds were added when the agar had cooled to approximately $55^{\circ} \mathrm{C}$ after autoclaving.

Mycelial plugs of the individual isolates were removed from the advancing edge of a water agar culture with a 5-mm cork borer and inverted onto the center of the 15-cm-diameter dishes. The petri dishes were randomly placed under UV and white fluorescent light banks with a 12-h photoperiod (14) in a room at $21 \pm 5^{\circ} \mathrm{C}$. At 24,48 , and $72 \mathrm{~h}$ after placing the plug on the agar, the longest and shortest colony diameters were measured using a dissecting microscope ( $\times 12.6$ magnification).

The six concentrations were tested in separate experiments of three concentrations each. Each experiment included the corresponding unamended media, $2 \%$ water and $2 \%$ dextrose agar, as the controls. Radial growth over each 48-h interval between measurements was calculated from the mean of the measurements of each dish. Each petri dish was a statistical unit (replicate). There were three replications of each compound concentration on each of the two agar media in each experiment in a randomized complete block design where experimental repeats were blocks. The experiment was conducted twice.
Rate of hyphal extension (millimeters per hour) was calculated for the period between 24 and $72 \mathrm{~h}$ based on average measurements. The three lower and three higher concentrations were analyzed separately. Significance of differences in rate of hyphal extension among treatment main effects (isolate, media, and compound concentration) and their interactions were tested by analysis of variance (ANOVA) using the general linear model in MINITAB (release 12; MINITAB, Inc., State College, PA).

Spore germination. Ascospore and macroconidial germination of the three $F$. graminearum isolates was evaluated on plain glass microscope slides covered with a $10-, 100-$, and $1,000-\mu \mathrm{M}$ layer of choline- or betaine-amended $2 \%$ water and dextrose agar as described previously (9). Three 1- $\mu$ l drops of sterile water containing approximately 20 ascospores or macroconidia were placed on the slide. Slides then were placed onto the surface of sterile $2 \%$ water agar dishes to maintain adequate moisture. The dishes were randomly placed under the light bank previously described.

Each drop of spore suspension on a slide was a subunit. Each slide for each compound-amended agar type was a replicate, and there were two replicates per experiment. The experiment was conducted twice. The numbers of germinated spores were counted using a dissecting microscope ( $\times 12.6$ magnification) 2, 4, 6, 8 , and $24 \mathrm{~h}$ after placing the spore suspensions on the agar coated slides. A spore was considered germinated when the germ tube was clearly discernible. The slides were not removed from the petri dishes during spore counts.

Significant differences in percentage of germinated spores among treatment main effects (isolate, media, and compound concentration) and interactions were tested by ANOVA for a completely randomized block design where blocks were experi-

Table 1. Mean severity and incidence of Fusarium head blight on nine genotypes selected from the 1999 uniform winter wheat screening nursery ${ }^{\mathrm{y}}$

\begin{tabular}{lccc}
\hline Genotype & Incidence (\%) & Severity $(\%)$ & Resistance $^{\mathbf{z}}$ \\
\hline Ernie & 36.1 & 8.6 & PR \\
IL94-1909 & 28.3 & 9.1 & PR \\
OH552 & 53.9 & 11.8 & PR \\
Goldfield & 38.1 & 14.1 & PR \\
Freedom & 44.5 & 15.5 & PR \\
OH609 & 35.4 & 15.9 & PR \\
IL95-4162 & 34.5 & 17.6 & PR \\
Patterson & 49.5 & 24.6 & S \\
2545 & 61.0 & 19.9 & S \\
LSD & 13.3 & 6.4 & $\ldots$ \\
\hline
\end{tabular}

${ }^{y}$ Nurseries were evaluated at six locations across the United States and one location in Ontario, Canada. Incidence is the mean percentage of spikes with at least one symptomatic spikelet. Severity is the mean percentage symptomatic spikelets per spike.

${ }^{\mathrm{z}}$ Relative reaction compared with 2545 based on incidence and severity assessments, where PR = partially resistant and $S=$ susceptible. Classification based on cultivar means from incidence or severity significantly different (for PR classification) or not significantly different (for S classification) from the susceptible cultivar 2545 for resistance according to Fisher's protected least significant difference (LSD) $(P \leq 0.05)$. mental repeats using the general linear model in MINITAB. Data for the two different spore types were analyzed separately.

Floral compound extracts. Nine wheat genotypes were selected to evaluate the effect of floral compound extracts on the growth of $F$. graminearum. Selection was based on differences in mean Fusarium head blight severity and incidence from the 1999 uniform winter wheat Fusarium head blight screening nursery (6; Table 1). Mean incidence and severity were based on seven field locations across six states in the United States and one nursery in Ontario, Canada. Seed harvested from the nursery planted in Wooster, $\mathrm{OH}$ was used for this experiment. The seed was stored at $4.5^{\circ} \mathrm{C}$ under low relative humidity until use.

Seed were germinated and placed in a growth chamber at $3.5^{\circ} \mathrm{C}$ with an 8 -h photoperiod for approximately 65 days for vernalization. Four seedlings for each genotype were transplanted into individual $15.2-\mathrm{cm}$ pots containing autoclaved Wooster silt-loam soil and placed by genotype on benches in a $20.0 \pm 3.0^{\circ} \mathrm{C}$ greenhouse with a 12-h photoperiod of supplemental lighting. Standard greenhouse conditions were used to produce healthy plants (9).

Each pot containing four plants per genotype was considered a statistical unit (replicate). There were six replicates per block. Each of three temporally repeated experiments was a block. The blocks were separated by a month in planting date.

Entire spikes were collected when at least one floret had extruded anthers. Fifteen spikes randomly collected from all the replicates of a genotype in a block were placed in a plastic bag and stored in a freezer at $-10^{\circ} \mathrm{C}$ until dissection. From each of the 15 spikes, 11 anthers, two paleas, and two lemmas were randomly collected. A greater number of anthers were harvested from the spikes because of the smaller size of anthers compared with lemmas and paleas. The individual floral tissues collected from the 15 spikes of each block were combined to form the floral tissue sample for each genotype. Therefore, there were three floral tissue samples for each of three blocks.

During dissection, the lemma, palea, and anthers were removed with sterile, finetipped forceps and placed in separate 1.5$\mathrm{ml}$ Eppendorf tubes containing $0.5 \mathrm{ml}$ of $100 \%$ methanol and stored in a freezer at $-10^{\circ} \mathrm{C}$. Samples were stored in $100 \%$ methanol to sterilize sampled tissues. The Eppendorf tubes were opened and placed in an air bench to evaporate the methanol and the samples were rehydrated with $1 \mathrm{ml}$ of sterile water. The samples were frozen and thawed once before being ground with a polypropylene pellet pestle (Kontes Glassware, Vineland, NJ) in Eppendorf tubes. Tubes were centrifuged and the supernatant transferred to fresh tubes. Tissue maceration and extraction was re- 
peated twice and the supernatants from each sample were pooled.

A $2.5-\mathrm{ml}$ aliquot of each pooled supernatant was mixed with $2.5 \mathrm{ml}$ of $4 \%$ water agar in sterilized test tubes in a $55^{\circ} \mathrm{C}$ water bath. The amended agar $(5 \mathrm{ml})$ was vortexed and then poured into $5-\mathrm{cm}$-diameter petri dishes. Plugs of two $F$. graminearum isolates were removed from the advancing edges of water agar cultures with a $5-\mathrm{mm}$ cork borer and inverted on the surface of the amended agar with individual isolate plugs on opposite sides of the petri dish. Hyphal growth from the edge of each plug was measured after 24, 48, and $72 \mathrm{~h}$. Two measurements for each isolate were averaged per tissue sample per block per genotype.

Rate of hyphal extension (millimeters per hour) was calculated for the period between 24 and $72 \mathrm{~h}$. The different floral tissues were analyzed separately. Percentage of increased growth compared with the control (unamended water agar) was calculated for each floral part extract. Significance of differences in the percentage of increased growth compared with the control among treatment main effects (wheat genotype and $F$. graminearum isolate) and interaction was tested by ANOVA conducted using the general linear model in MINITAB.

\section{RESULTS}

Hyphal extension. The three $F$. graminearum isolates had significantly different $(P=0.05)$ linear growth rates on amended agar plates over the time period measured, but growth rate of an isolate was constant across repeats of experiments. The three isolates had a mean radial growth rate of 0.35 to $0.64 \mathrm{~mm} / \mathrm{h}$ on water agar, which was significantly different $(P<$ $0.0001)$ from the mean radial growth rate of 0.35 to $0.54 \mathrm{~mm} / \mathrm{h}$ on dextrose agar. Choline induced a relatively small but significant $(P=0.05)$ increase in radial growth of the three isolates on water agar, but not on dextrose agar, at concentrations from 0.01 to $1.0 \mu \mathrm{M}$ compared with the unamended control after $72 \mathrm{~h}$ (Fig. 1A). However, this small effect was not observed at concentrations ranging from 10 to $1,000 \mu \mathrm{M}$ (Fig. 2A).

The three isolates also had a relatively small but significant $(P=0.05)$ increase in radial growth on water agar amended with betaine, but not on amended dextrose agar, at concentrations from 0.01 to $1.0 \mu \mathrm{M}$ compared with the unamended control after $72 \mathrm{~h}$ (Fig. 1B). Betaine did not significantly affect radial growth at concentrations of 10 to $100 \mu \mathrm{M}$, although there was a reduction of hyphal growth of all isolates at the 1,000$\mu \mathrm{M}$ concentration compared with the unamended control of both agars (Fig. 2B).

Likewise, the equimolar mixture of choline and betaine slightly, but significantly, $(P=0.05)$ increased the radial growth of the isolates at concentrations ranging from
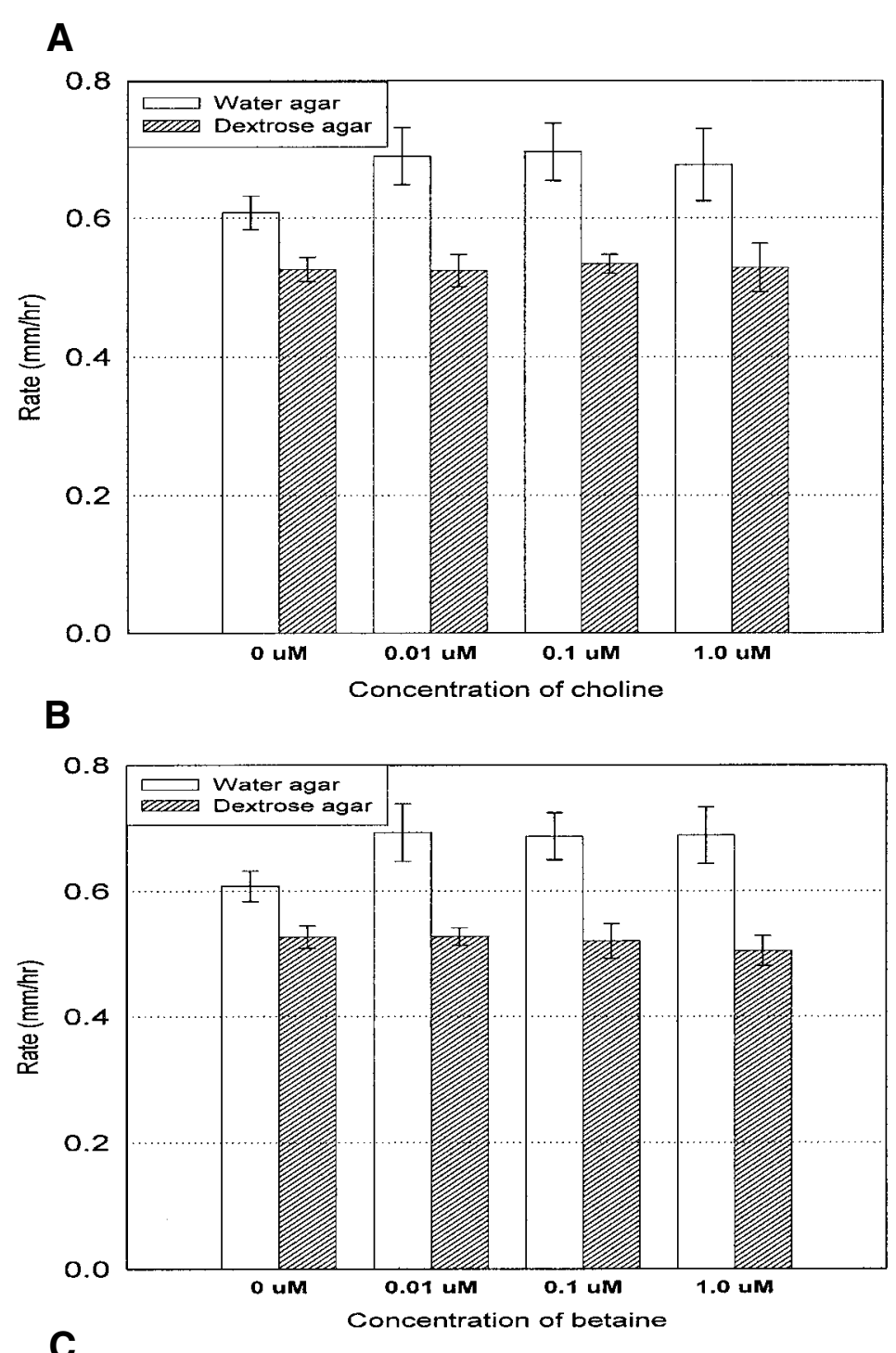

C

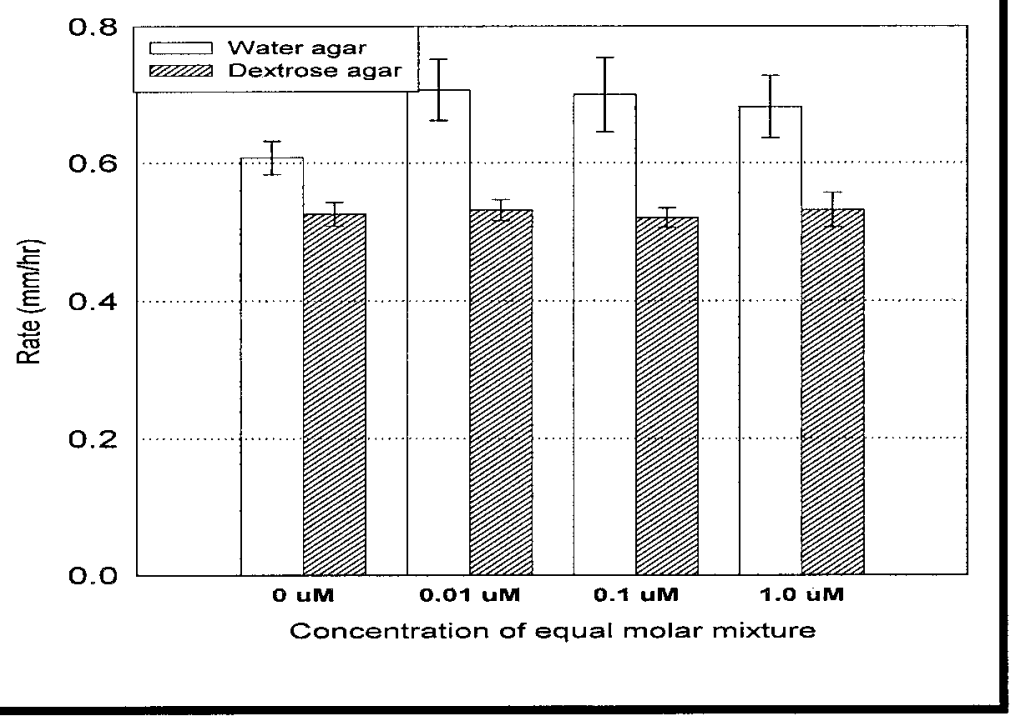

Fig. 1. Mean radial growth rate of three Fusarium graminearum isolates over a 72-h period on water and dextrose agar amended with $\mathbf{A}$, choline; $\mathbf{B}$, betaine; and $\mathbf{C}$, equimolar mixture of choline and betaine at compound concentrations ranging from 0 (unamended control) to $1.0 \mu \mathrm{M}$. Data presented are the means from three experiments (means were combined across experiments, with each experiment having three replicate plates per agar type per compound at each concentration). Bar indicates one standard deviation. 
0.01 to $1.0 \mu \mathrm{M}$ (Fig. 1C). The equimolar concentrations of choline and betaine significantly $(P \leq 0.01)$ reduced hyphal growth at the $1,000-\mu \mathrm{M}$ concentration compared with the unamended control of both agars, but had little effect on hyphal growth at concentrations ranging from 10 to $100 \mu \mathrm{M}$ (Fig. 2C).

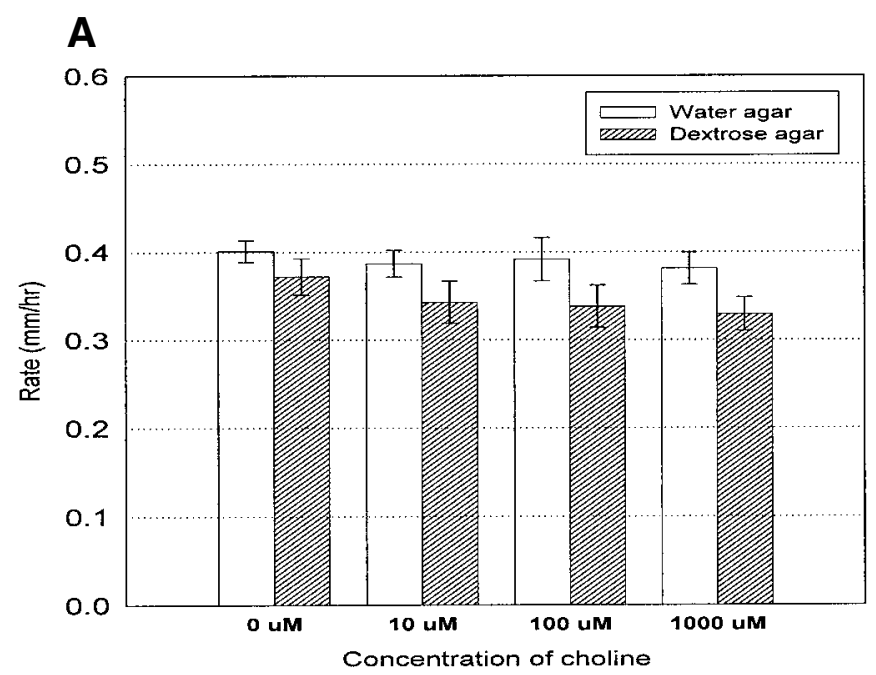

\section{B}

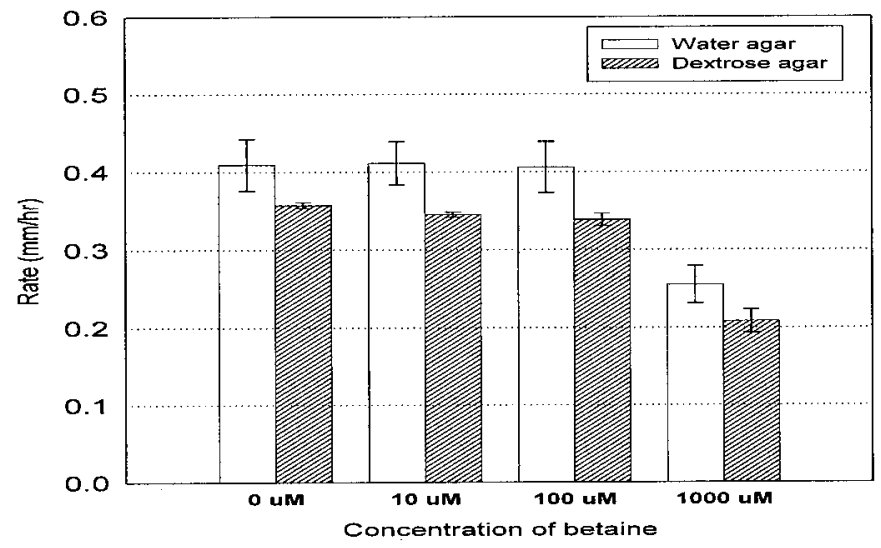

\section{C}

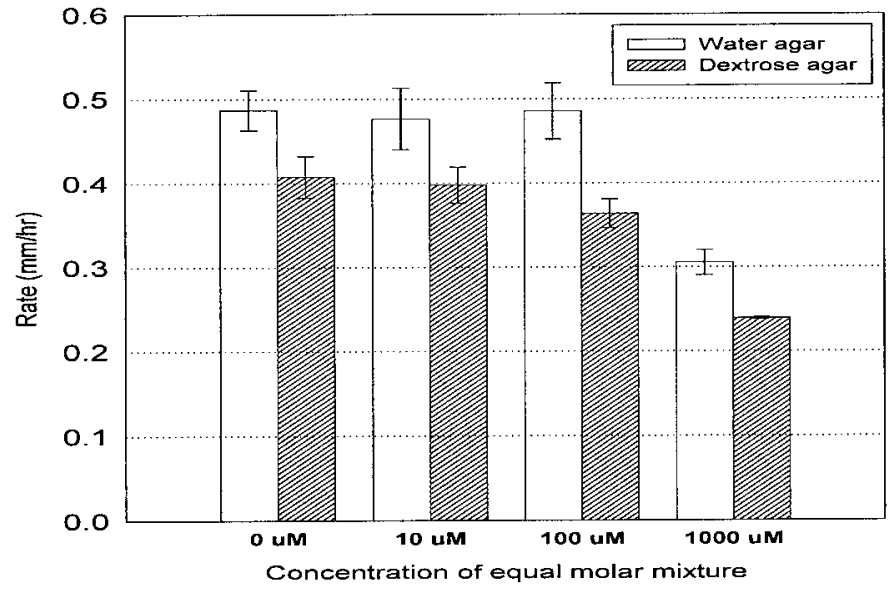

Fig. 2. Mean radial growth rate of three Fusarium graminearum isolates over a 72-h period on water and dextrose agar amended with $\mathbf{A}$, choline; $\mathbf{B}$, betaine; and $\mathbf{C}$, equimolar mixture of choline and betaine at compound concentrations ranging from 0 (unamended control) to 1,000 $\mu \mathrm{M}$. Data presented are the means from three experiments (means were combined across experiments, with each experiment having three replicate plates per agar type per compound at each concentration). Bar indicates one standard deviation.

Spore germination. Ascospores and macroconidia germinated readily on unamended water agar- and dextrose agarcoated glass slides with $99 \%$ germination within $24 \mathrm{~h}$. Germination of ascospores and macroconidia was not significantly $(P$ $=0.05)$ affected by 10-, 100-, and 1,000$\mu \mathrm{M}$ concentrations of choline, betaine, or an equimolar mixture when compared with the unamended control slides over a 24-h period (data not shown).

Floral compound extracts. Both isolates of $F$. graminearum used to evaluate growth on tissue extract-amended agar grew readily on water agar, but at significantly different rates $(P=0.05)$, with an average of $0.3 \mathrm{~mm} / \mathrm{h}$. Hyphal growth of the two $F$. graminearum isolates was significantly increased $(P=0.05)$ by anther and palea extracts of all genotypes when compared with the unamended control (Fig. 3A and B). The lemma extracts also significantly increased $(P=0.05)$ hyphal growth for both isolates except when placed on the agar amended with extracts from the genotypes Goldfield (partially resistant) and 2545 (susceptible) (Fig. 3C). The growth stimulation by floral extracts was not consistent with resistance reactions of the genotypes as determined by disease assessments in the field (Table 1).

\section{DISCUSSION}

The effects of choline and betaine on macroconidial germination have been previously studied $(15,24)$. In both studies, concentrations of choline, betaine, and an equimolar mixture of the two compounds ranging from $0.1 \mu \mathrm{M}$ to $1 \times 10^{4} \mu \mathrm{M}$ in amended Vogel's salt solution and $2 \%$ sucrose agar had no effect on germination of macroconidia $(15,25)$. In this study, employing different protocols and simple media, these results were confirmed over a compound concentration range of 0.01 to $1,000 \mu \mathrm{M}$. Ascospore germination in the presence of choline and betaine also was examined in our study. Germination of ascospores also was found to not be increased by the presence of choline or betaine. It was concluded that, regardless of the nutrient environment ascospores or macroconidia were exposed to, choline and betaine had no effect on germination.

Previous researchers have used liquid and solid media with Vogel's salt solution modified with either $2 \%$ sucrose or glucose and amended with choline chloride or betaine hydrochloride to determine the effects of these compounds on hyphal growth of $F$. graminearum in vitro $(15,24,27)$. Strange and Smith (25) reported that choline in concentrations as low as $0.01 \mu \mathrm{M}$ caused a small, but statistically significant enhancement of $F$. graminearum radial growth on water agar. Our results concur with these results and indicate that higher concentrations $(\geq 10$ $\mu \mathrm{M})$ of choline on agar modified with dextrose did not affect radial growth when 
compared with the unamended control $(15,25,27)$. Betaine and equimolar mixtures of choline and betaine had no effect on $F$. graminearum radial growth at low concentrations; however, in this and previ- ous studies (15), betaine inhibited or reduced growth at higher $(\geq 1,000 \mu \mathrm{M})$ concentrations. We used different protocols than these previous studies to examine the response of $F$. graminearum to choline and

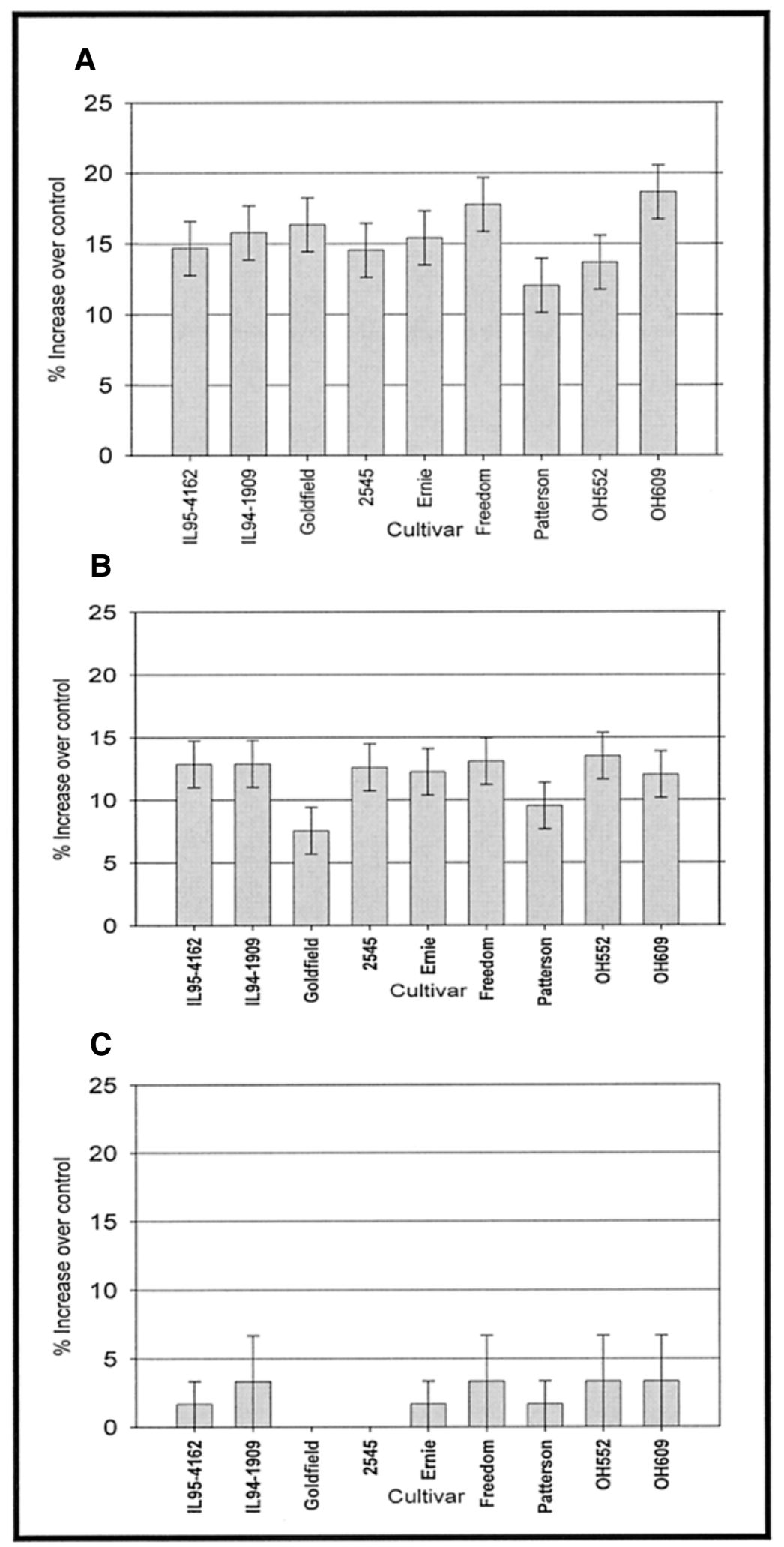

Fig. 3. Mean percentage increase in growth $(\mathrm{mm} / \mathrm{hr})$ over the water agar control of two Fusarium graminearum isolates grown on medium amended with floral tissue extracts from nine wheat genotypes. A, Anther extracts (least significant difference $[\mathrm{LSD}]=7.49$ ); $\mathbf{B}$, palea extracts $(\mathrm{LSD}=5.97$ ); and $\mathbf{C}$, lemma extracts $(\mathrm{LSD}=\mathrm{NS})$. Data are the means of two isolates of $F$. graminearum on three replicate plates per floral tissue of each cultivar. Bar indicates one standard deviation.

betaine to determine the validity of previously reported results. We concluded that these compounds have no or, at the most, only a minor role in colonization of floral tissue by $F$. graminearum. It was not clear why the higher concentrations of betaine reduced growth compared with the unamended control, whereas lower concentrations of betaine increased growth in this study. We did observe increased branching of hyphae at higher betaine concentration treatments. It may be that higher concentrations of betaine perturb other biological processes in $F$. graminearum, such as osmoregulation (7), and negatively affect growth.

Strange et al. (25) found that anther extracts highly enhanced fungal growth and colonization when added to inoculum suspensions. Other researchers found that anther extracts from a cultivar with a resistant reaction to Fusarium head blight significantly enhanced radial hyphal growth compared with the control and anther extracts from a susceptible cultivar (15). In our study, anther extracts from nine genotypes with various levels of resistance expressed in the field enhanced fungal growth to a greater extent than palea or lemma extracts. It was assumed that the higher concentration of sugars in anthers compared with the other floral structures caused the enhancement of growth. Additionally, there appeared to be no association between genotype resistance reaction in the field and enhancement of fungal growth by any of the extracts from floral structures.

Results from this study indicate that choline and betaine have no effects on germination of macroconidia or ascospores at the concentrations tested. These compounds also appear to have biologically insignificant effects on subsequent growth of $F$. graminearum at levels occurring in floral tissues. Furthermore, our results from tests using genotypes with a broad genetic background indicate that constitutive compounds in floral structures do not differ greatly among genotypes in their ability to influence hyphal growth and probably have no substantial role in resistance to $F$. graminearum. Therefore, it is concluded that preformed floral compounds are not significant in the resistance response of wheat when infected by $F$. graminearum.

\section{ACKNOWLEDGMENTS}

We thank A. L. Johnston for technical assistance.

\section{LITERATURE CITED}

1. Andersen, A. L. 1948. The development of Gibberella zeae head blight of wheat. Phytopathology 38:595-611.

2. Arseniuk, E., Foremska, E., Goral, T., and Chelkowski, J. 1999. Fusarium head blight reactions and accumulation of deoxynivalenol (DON) and some of its derivatives in kernels of wheat, triticale, and rye. J. Phytopathol. 147:577-590. 
3. Arthur, J. C. 1891. Wheat scab. Indiana Agric. Exp. State Bull. 36:129-138.

4. Bai, G.-H., and Shaner, G. 1994. Scab of wheat: Prospects for control. Plant Dis. 78:760-766.

5. Bai, G.-H., and Shaner, G. 1996. Variation in Fusarium graminearum and cultivar resistance to wheat scab. Plant Dis. 80:975-979.

6. Campbell, K. G., and Franchino, B. 1999. Uniform winter wheat Fusarium head blight screening nursery 1999 nursery report. Ohio State Univ. Hortic. Crop Sci. Ser. 690.

7. Cosquer, A., Pichereau, V., Pocard, J.-A., Minet, J., Cormier, M., and Bernard, T. 1999. Nanomolar levels of dimethylsulfoniopropionate, dimethylsulfonioacetate, and glycine betaine are sufficient to confer osmoprotection to Escherichia coli. Appl. Environ. Microbiol. 65:3304-3311.

8. Dill-Macky, R., and Jones, R. K. 2000. The effect of previous crop residues and tillage on Fusarium head blight of wheat. Plant Dis. 84:71-76.

9. Engle, J. S. 2002. Evaluation and characterization of resistance to Fusarium graminearum in winter wheat. M.Sc. thesis, The Ohio State University, Columbus.

10. Martin, R. A., MacLeod, J. A., and Caldwell, C. 1991. Influences of production inputs on incidence of infection by Fusarium species on cereal seed. Plant Dis. 75:784-788.

11. McMullen, M., Jones, R., and Gallenberg, D. 1997. Scab of wheat and barely: a re-emerging disease of devastating impact. Plant Dis. 81:1340-1348.

12. Mills, J. T. 1982. Development of fusaria and fusariotoxins on cereal grains in storage. Can. J. Plant Pathol. 4:217-218.

13. Milus, E. A., and Parsons, C. E. 1994. Evaluation of foliar fungicides for controlling Fusarium head blight of wheat. Plant Dis. 78:697699.

14. Nelson, P. E., Toussoun, T. A., and Marasas, W. F. O. 1983. Fusarium Species: An Illustrated Manual for Identification. Pennsylvania State University, University Park.

15. Nkongolo, K. K., Dostaler, D., and Couture, L. 1993. Effet de la betaine, de la choline et d'extraits d'antheres du ble sur la croissance du Fusarium graminearum. Can. J. Plant Pathol. 15:81-84.

16. Parry, D. W., Jenkinson P., and Mcleod L. 1995. Fusarium ear blight (scab) in small grain cereals-a review. Plant Pathol. 44:207-238.

17. Paulitz, T. C. 1996. Diurnal release of ascospores by Gibberella zeae in inoculated wheat plots. Plant Dis. 80:674-678.

18. Pearce, R. B., Strange, R. N., and Smith, H. 1976. Glycinebetaine and choline in wheat: distribution and relation to infection by Fusarium graminearum. Phytochemistry 15:953-954.

19. Pugh, G. W., Johann, H., and Dickson, J. G. 1933. Factors affecting infection of wheat heads by Gibberella saubinetii. J. Agric. Res. 46:771-797.

20. Robson, G. D., Best, L. C., Wiebe, M. G., and
Trinci, A. P. J. 1992. Choline transport in Fusarium graminearum A3/5. FEMS Microbiol. Lett. 92:247-252.

21. Robson, G. D., Wiebe, M. G., and Trinci, A. P. J. 1994. Betaine transport in Fusarium graminearum. Mycol. Res. 98:176-178.

22. Snijders, C. H. A. 1990. Genetic variation for resistance to Fusarium head blight in bread wheat. Euphytica 50:171-179.

23. Strange, R. N., Majer, J. R., and Smith, H. 1974. The isolation and identification of choline and betaine as the two major components in anthers and wheat germ that stimulate Fusarium graminearum in vitro. Physiol. Plant Pathol. 4:277-290.

24. Strange, R. N., and Smith, H. 1971. A fungal growth stimulant in anthers which predisposes wheat to attack by Fusarium graminearum. Physiol. Plant Pathol. 1:141-150.

25. Strange, R. N., and Smith, H. 1978. Specificity of choline and betaine as stimulants of Fusarium graminearum. Trans. Br. Mycol. Soc 70:187-192.

26. Strange, R. N., Smith, H., and Majer, J. R. 1972. Choline, one of two fungal growth stimulants in anthers responsible for the susceptibility of wheat to Fusarium graminearum. Nature 238:103-104.

27. Wiebe, M. G., Robson, G. D., and Trinci, A. P. J. 1989. Effect of choline on the morphology, growth and phospholipid composition of Fusarium graminearum. J. Gen. Microbiol. $135: 2155-2162$. 